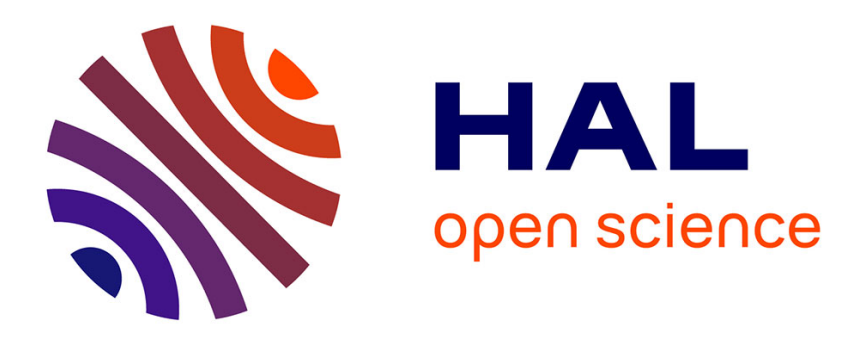

\title{
Les effets sociaux de la pensée politique sur l'insertion - L'exemple du R.M.I. "
}

\author{
Alain Thalineau
}

\section{To cite this version:}

Alain Thalineau. Les effets sociaux de la pensée politique sur l'insertion - L'exemple du R.M.I. ". L'Homme et la Société, 1997, n²125. hal-01415251

\section{HAL Id: hal-01415251 \\ https://hal.science/hal-01415251}

Submitted on 12 Dec 2016

HAL is a multi-disciplinary open access archive for the deposit and dissemination of scientific research documents, whether they are published or not. The documents may come from teaching and research institutions in France or abroad, or from public or private research centers.
L'archive ouverte pluridisciplinaire HAL, est destinée au dépôt et à la diffusion de documents scientifiques de niveau recherche, publiés ou non, émanant des établissements d'enseignement et de recherche français ou étrangers, des laboratoires publics ou privés. 


\title{
Les effets sociaux de la pensée politique sur l'insertion.
}

\author{
-L'exemple du R.M.I.-
}

A. Thalineau

Lorsque les chercheurs en sciences sociales prennent comme thème de recherche les pratiques de lutte contre la pauvreté, les faits se présentant à eux sous la forme de "problèmes sociaux" ne se laissent pas aisément appréhender. La complexité du phénomène à étudier est en effet accentuée par la difficulté à établir, en tant que sociologue ou économiste, la distance nécessaire. D'une part, lorsque l'on se trouve quotidiennement en face à face avec des personnes en quête (de travail, de ressources, de logement), dans le métro, aux feux des carrefours routiers, sur les trottoirs, le discours scientifique a parfois quelque peine à prendre le recul indispensable. D'autre part, le chercheur doit sans cesse effectuer une rupture avec les discours des hommes politiques ou des journalistes qui imposent leurs visions du monde et qui sont parfois les financeurs des recherches. L'un des moyens pour parvenir à comprendre le sens des pratiques de lutte contre la pauvreté est d'inclure ces discours dans l'objet étudié. Cette contribution se situe dans cette optique. La réflexion a pour finalité de comprendre les liens entre les discours politiques et les pratiques sur l'insertion, et cela plus précisément dans le cadre du R.M.I.. Il s'agit d'étudier pourquoi la loi de 88 , révisée en 92, votée à une très large majorité, a pu être appliquée en prenant pour but le retour progressif à l'emploi, alors que ce but est reconnu par ailleurs comme étant impossible à atteindre. 
Pour comprendre pourquoi ce paradoxe n'est qu'apparent, le recours à l'histoire est utile. Par cette prise de distance historique, la pensée politique sur l'insertion présente dans la loi du R.M.I. apparaît comme la résultante des rapports sociaux entre les détenteurs du pouvoir économique et politique et les populations dans l'impossibilité de subvenir par eux-mêmes à leurs propres besoins, cela depuis l'avènement du capitalisme. Elle est une logique qui, autour de la notion de contrat, articule la responsabilité individuelle et la responsabilité collective de telle façon qu'il devient possible de reconnaître conjointement que "la véritable insertion consiste à trouver un emploi"1 et qu'il n'y a plus assez d'emplois pour occuper l'ensemble de la population active. Après une description des principes qui constituent cette logique, il s'agit de présenter en quoi elle prend tout son sens dans la relation entre les bénéficiaires ${ }^{2}$ et les travailleurs sociaux au sein du champ d'exécution de la politique sociale du R.M.I..

\section{Les fondements de la logique d'insertion : la primauté de la responsabilité individuelle sur}

\section{la responsabilité collective.}

La logique de la pensée politique sur l'insertion présente dans les débats à l'Assemblée Nationale articule les notions de "travail", de responsabilités "individuelle" et "collective", et de "contrat" pour parfaire le système visant à limiter l'exclusion sociale. Si l'articulation proposée dans les textes de loi ne suscite pas d'importantes controverses lors des débats, c'est en raison du fait qu'elle est un construit conforme aux représentations dominantes de ce qu'il faut faire pour enrayer le phénomène. Ces représentations sont le produit de l'histoire de la lutte contre la pauvreté depuis l'émergence d'un Etat cherchant à intervenir en matière économique et sociale dès la fin du XIVème siècle. Les travaux de recherche déjà réalisés sur cette question ont montré comment les interventions à l'égard des populations

\footnotetext{
$1 \quad$ Jean-Piere Sueur, Parti socialiste, débat à l'Assemblée Nationale du 11.10.88.

2 En choisissant ce terme pour désigner la population entrant en relation avec un travailleur social dans le cadre du R.M.I. et en le mettant en italiques, l'intention est de nommer la catégorie administrative telle qu'elle est désignée par les agents chargés de la mise en application de la loi, tout en indiquant que cette désignation contient des présupposés liés à la logique du champ à analyser ; voir infra p 6 .
} 
étiquetées selon les époques comme "gueux, "mendiants", "pauvres", "pauvres travailleurs"³, ont été l'objet de débats entre :

- ceux qui évoquent la responsabilité individuelle du "pauvre" (responsable de son sort) et la responsabilité individuelle du "riche" pour éradiquer la pauvreté,

- ceux qui reconnaissent une responsabilité collective à l'égard des "pauvres" incapables de subvenir par eux-mêmes à leur propre besoin ("les victimes de leur sort") et qui affirment la nécessité d'une répression à l'égard des "oisifs" ("les responsables de leur sort"),

- et enfin ceux qui considèrent l'Etat comme le seul responsable, son rôle étant de garantir à tous les moyens de vivre.

Sans réécrire cette histoire ${ }^{4}$, on peut cependant souligner que ces points de vue divergents, correspondant à des positions sociales distinctes (protestants/catholiques ; libéraux, catholiques sociaux, socialistes, républicains...), trouvent un compromis à la fin du XIXème siècle par une nouvelle articulation des responsabilités autour de la notion de solidarité. Cette articulation constitue la structure historique de la logique d'insertion présente dans la loi sur le R.M.I..

\section{1- La solidarité comme réponse à la question sociale.}

A la fin du XIXème siècle, dans un contexte de transformations socio-économiques, l'idée de solidarité qui était présente au sein de la technologie assurantielle libérale $\mathrm{e}^{5}$ prend un autre sens. Comme le souligne Pierre Rosanvallon, la solidarité libérale devient républicaine dans le contexte de la révolution pastorienne qui montre l'existence permanente de relations d'interdépendance entre les agents sociaux : "En révélant le processus d'action microbien, Pasteur donne un fondement nouveau à la perception de l'interaction sociale. Il montre que les individus ne peuvent être appréhendés comme des monades isolées

3 La liste n'est pas exhaustive tant les appellations ont été nombreuses.

$4 \quad$ Voir par exemple l'ouvrage de Robert Castel, Les métamorphoses de la question sociale. Une chronique du salariat, Paris, Fayard, 1995,

5 François Ewald, L'Etat Providence, Paris, Ed. Grasset, 1986, pp 184-185. 
et que des liens invisibles - ceux du microbe - les relient à leur insu ${ }^{\prime \prime}$. La révolution pastorienne montre qu'il n'est pas concevable de représenter les individus en dehors de toute relation d'interdépendance et que les maladies ont des répercussions sociales. Il ne s'agit plus d'une solidarité nécessaire pour se protéger des risques éventuels, mais d'une solidarité de fait. Le terme de solidarité devient incontournable. Il est rapidement transposé de la biologie à la sociologie. Emile Durkheim lui a donné toute sa force théorique dans sa thèse "De la division du travail social" parue en 1893. La "solidarité" est présentée comme le constituant du lien social entre les individus vivant en société et permet de sortir de la fiction philosophique du contrat social.

Cette approche durkheimienne trouve un écho favorable dans le mouvement "solidariste" animé par Léon Bourgeois, radical socialiste, ministre du travail en 1911. Dans son ouvrage "solidarité", écrit en 1896, il indique dès les premières pages sa volonté de trouver une autre orientation qui ne soit pas un intermédiaire entre le libéralisme et le socialisme, mais qui soit un dépassement. Son raisonnement, construit autour des termes de solidarité, de dette et de quasi-contrat ${ }^{7}$, permet d'articuler de façon spécifique les responsabilités individuelle et collective en matière de lutte contre la pauvreté.

L'homme de Léon Bourgeois doit son existence au travail accompli par ses proches et par les générations passées. Il doit oeuvrer au développement pour ne pas détruire les richesses communes dont il hérite. Il a une dette avant même d'avoir un droit : "L'homme naît débiteur de la société ${ }^{8}$. Pour recevoir, les individus sociaux doivent donner. L'échange qui en découle, n'est pas nécessairement le produit d'un accord explicite ; il vise cependant à l'équilibre des avantages et inconvénients entre les protagonistes. Cet échange constitue la matière du quasi-contrat d'association. La société, dont l'Etat est représenté comme l'instrument, doit évaluer la juste répartition de "l'actif et du passif social ${ }^{9} "$.

$6 \quad$ Pierre Rosanvallon, 1990, L'Etat en France, Paris, Ed. du Seuil, Coll. U. H, p 130.

$7 \quad$ Voir Jacques Donzelot, L'invention du social, Paris, Fayard, 1984, 263 p

8 Léon Bourgeois, 1931, Solidarité, Paris, Lib. Armand Colin, p 54 (réédition de textes de l'auteur parus entre 1896 et 1909).

9 Léon Bourgeois, op.cit., p 63. 
L'intervention de l'Etat apparaît alors sous un nouvel aspect. Elle n'est plus, comme le craignent les libéraux, une atteinte à la liberté individuelle ; elle n'est pas illimitée, comme le souhaitaient les socialistes révolutionnaires du XIXème siècle. Le lien social étant au centre de la société ainsi définie, l'Etat apparaît comme un régulateur qui se doit de garantir la disparition des inégalités de condition ; par l'impôt, les individus payent leur dette à l'égard de la société qui doit en retour supporter la charge des risques sociaux qu'elle génère $^{10}$. Ainsi, la responsabilité collective n'exclut pas la responsabilité individuelle. Celle-ci reste première. Le "pauvre" peut devenir un bénéficiaire de droits sociaux à condition d'avoir respecté ce "quasicontrat" qui consiste à payer sa dette, principalement en travaillant.

La solidarité est indissociable de l'idée de progrès par le développement de l'économie de marché qui nécessite un effort individuel et collectif pour un partage plus juste des fruits de ce progrès. Lorsque la pauvreté revient au premier rang des préoccupations politiques, les solutions envisagées se construisent à partir des principes du solidarisme. La loi sur le Revenu Minimum d'Insertion est pensée en ces termes.

\subsection{Le R.M.I., une synthèse historique des principes de lutte contre la pauvreté}

Dans une période de l'histoire du capitalisme caractérisée par la fin du mythe du plein emploi, la question de la pauvreté liée à l'absence d'emploi trouve ses réponses dans le solidarisme :

La loi sur le R.M.I. s'inscrit dans le prolongement de l'Etat providence tout en y intégrant sa critique. La responsabilité collective n'est plus engagée uniquement par l'Etat; les agents ou corps d'agents situés au plus près des populations ont un rôle essentiel.

La responsabilité du bénéficiaire est placée au premier plan dans la réussite de l'insertion. Cette centration sur l'individu responsable de son sort, dépendante des principes même du système capitaliste, est ce qui permet de sortir du paradoxe : il n'y a pas d'emploi pour tous mais tous ne sont pas capables d'avoir un emploi ; le rôle des pouvoirs publics est de les aider à devenir "employables" en réalisant avec eux un "contrat d'insertion".

10

Id., p 238. 


\section{A- Les solidarités locales face à la solidarité étatique.}

Le R.M.I. est financé par l'impôt et non par des cotisations salariales et patronales, tout en étant géré par les Caisses d'Allocations Familiales ou de Mutualité Sociale Agricole. L'Etat maintient sa position de régulateur socio-économique qu'il a acquise pendant la période dite des "trente Glorieuses". Le patronat n'est donc pas directement impliqué dans le financement de cette loi. Même si le R.M.I. concerne des travailleurs potentiels exclus du champ économique, il n'est pas une assurance chômage qui imposerait une quelconque obligation financière au patronat. Seuls les députés communistes ont souhaité le rétablissement de l'impôt sur les grandes fortunes pour financer le R.M.I..

La centralisation par l'impôt et la décentralisation de la gestion répondent à la volonté respective de maintenir l'Etat dans sa fonction protectrice et de développer les solidarités locales. L'expression de cette responsabilité collective ne se limite pas seulement à financer et à organiser des actions visant à aider les agents relevant du R.M.I. sur un territoire, le département, subdivisé en secteurs correspondant aux aires des Commissions Locales d'Insertion (C.L.I.). Elle consiste également à engager un suivi individuel des allocataires en vue de les soutenir dans leur engagement à sortir de "l'exclusion". Le contrat d'insertion symbolise la rencontre entre les deux responsabilités.

\section{B- Le contrat d'insertion : un support pédagogique.}

Les députés conçoivent le processus d'insertion comme un "accompagnement" de "l'exclu" afin de lui permettre de (re)construire des liens sociaux. "L'accompagnateur"11 est un travailleur social dont l'intervention est le complément indispensable au versement d'un revenu. Cette relation en face à face est conforme au modèle d'intervention à l'égard des "pauvres" qui s'est élaboré au cours du siècle. Le

\footnotetext{
11 Ce terme ne figure pas dans la loi de 88 mais dans celle de 92 dans le complément de l'article 12 concernant la mise en oeuvre du contrat d'insertion.
} 
travailleur social $^{12}$ est chargé d'apporter une aide psycho-éducative dans le cadre d'une relation duelle, dont la finalité est de rendre l'agent plus responsable :

"L'insertion doit tendre avant toute chose à développer les ressources individuelles des personnes, leur potentiel de créativité et une certaine autonomie personnelle ; elle doit aussi permettre de rétablir la communication entre ces personnes et leur environnement" (Guy Lordinot, P.S $S^{13}$, débat du 5.10.88, p 701).

"(...) Mme Cacheux (P.S.) a eu bien raison de souligner que, dans bien des cas, l'insertion commence par la recherche d'un traitement pour assurer l'équilibre physique de celui qui n'a pas pu s'insérer. Ainsi que je l'ai déjà dit, c'est souvent par la lutte contre l'alcoolisme que commence la réinsertion sociale" (Léonce Deprez, R.P.R ${ }^{14}$., débat du 11.10.88, $p$ 824).

Cet "accompagnement" individuel privilégiant l'interprétation psycho-sociale de l'exclusion, place au premier plan la responsabilité des bénéficiaires dans le processus d'insertion. L'articulation des deux responsabilités se réalise dans le cadre d'un quasi contrat social, le contrat d'insertion.

Celui-ci concrétise l'engagement des contractants dans leur devoir d'insertion et doit en permettre le contrôle :

"Il est donc bon d'inscrire dans la loi le devoir d'insertion pour l'individu, mais il est également nécessaire d'y faire figurer le devoir d'insertion pour la société, faute de quoi la notion même de contrat perdrait tout intérêt" (Jean-Pierre Sueur, P. S., débat du 5.10.88, p 694).

"Pour notre groupe, l'ardente obligation de l'insertion ne s'impose pas seulement aux bénéficiaires, mais à l'ensemble du corps social qui doit se mobiliser pour trouver des réponses adaptées à chaque personne. Cette obligation passe par l'élaboration d'un contrat

12 Selon le rapport de la Commission Nationale d'Evaluation présidée par Pierre Vanlerenberghe,il s'agit principalement d'assistants sociaux (Pierre Vanlerenberghe, R.M.I. le pari de l'insertion - Rapport de la commission nationale -, Paris, Ed. La documentation française 1992, T 1, p 346).

Parti Socialiste.

14 Rassemblement Pour la République. 
d'insertion (...). Il n'est pas question de subordonner le versement du R.M.I. à la signature dudit contrat, mais il faut pouvoir suspendre le versement de l'allocation si la personne ne respecte pas ses engagements." (Roselyne Bachelot, R.P.R., débat du 4.10.88, p 658).

Ainsi, le contrat a une double finalité ; il est le support d'une intervention psycho-éducative qui vise à établir chez l'individu son sens de la responsabilité consistant à s'engager dans des "actions d'insertion", qu'elles soient "sociales" ou "professionnelles", en vue de combler les manques ayant provoqué "l'exclusion" ; le contrat est aussi le support d'une intervention répressive lorsque les comportements de l'allocataire ne sont plus en conformité avec ce sens de la responsabilité. Seuls les députés communistes s'opposent à ce que la solidarité impose un devoir aux bénéficiaires :

"Pour vous le R.M.I. doit contribuer à une insertion. Comment y contribueraient les "petits boulots". Nous contestons bien évidemment ce point de vue. Le versement de l'allocation différentielle ne devrait souffrir d'aucune contrepartie. Ce devrait être seulement un élément de solidarité. Aussi sommes-nous inquiets de découvrir dans le projet de loi portant diverses mesures d'ordre social, des dispositions permettant à un employeur d'utiliser les services d'un bénéficiaire du R.M.I." (Muguette Jacquaint, P.C.F. ${ }^{15}$, débat du 21.11.88, $p$ 2588).

Les députés communistes ont associé l'exigence d'une contrepartie à un risque d'accroissement de l'exploitation, les bénéficiaires du R.M.I. pouvant être une "armée de réserve" pour le patronat. Ils se refusent à considérer les "exclus" comme des individus ayant une responsabilité dans leur situation. Ce point de vue n'est pas partagé par les autres groupes de députés. Selon eux, "le bénéficiaire" est dans un autre monde et le contrat a une fonction pédagogique :

"Assurer aux plus démunis des moyens de subsistance, mais tout faire pour leur permettre de retrouver une place dans $\underline{\text { notre }}^{16}$ société." (Roselyne Bachelot, R.P.R., débat du 4.10.88, $p$ 657).

15 Parti Communiste Français.

$16 \quad$ Souligné par nous. 
"Quant à nous élus de l'opposition, nous entendons montrer que nos objectifs sont différents et reposent avant tout sur la volonté de permettre à chaque individu de retrouver sa dignité d'homme en s'intégrant dans la société" (Elisabeth Hubert, R.P.R., débat du 5.10.88, $p$ 703).

"Ce que l'on demande dans le cadre du contrat d'insertion à celui qui entreprend, parfois après une longue période de mise à l'écart des liens sociaux ou de la vie professionnelle et productive, c'est de cheminer progressivement vers un but qui est son insertion professionnelle." (Jean-Michel Belorgey, P.S., débat du 11.10.88, p 828)

Le contrat symbolise un accord entre "la société" et "l'individu" pour permettre à des agents qui sont "sans place", "sans dignité", en dehors de la "société" pensée comme un tout dissociable de l'individu ${ }^{17}$, de passer dans la société de droit ${ }^{18}$. Ainsi, il y a désormais les agents qui n'ont pas contracté ("les exclus" ; "ceux qui sont rien"), ceux qui sont sous contrat ("les rmistes" ; "les bénéficiaires"), et ceux qui sont sortis et qui échappent à toute désignation stigmatisante. La période sous contrat constitue le temps de passage entre ces deux mondes. Plus qu'un rite de passage, la contractualisation est un rite d'institution, qui institue une différence entre ceux qui ne seront jamais concernés par ce rite et ceux qui le sont ${ }^{19}$; d'un côté, les agents ayant un travail ou un handicap justifiant une impossibilité à travailler, ayant des revenus supérieurs ou égaux au minimum, de l'autre côté les "exclus". Le contrat est donc un temps "pour cheminer progressivement vers un but", à savoir obtenir une place dans le système productif, le contrat de travail étant le prolongement théorique du contrat social que représente le R.M.I..

17 Pour plus de détail sur le sens de cette philosophie du contrat, voir Louis Dumont, Essais sur l'individualisme - Une perspective anthropologique sur l'idéologie moderne -, Paris, Ed. du Seuil, Coll. Esprit, 1983, $280 \mathrm{p}$

18 "Il (F. Mitterrand) a encore plus nettement précisé sa pensée au printemps à Montpellier en indiquant, ainsi que le rappelait Georgina Dufoix dans une lettre pastorale (sourires) qu'elle a fait parvenir à ses services au printemps : ce sont les droits qui sauvent les hommes et les femmes, car on ne les laisse plus à l'abandon dans des rapports de force où ils sont toujours perdants." (Jean-Michel Bellorgey, P.S., débat du 4.10.88, p 637).

19 Pierre Bourdieu,1982, "Les rites d'institution", in Actes de la recherche en sciences sociales, $\mathrm{N}^{\circ} 43$, Juin 1982, pp 58-69. 
Pour aller d'un contrat à l'autre, le projet ${ }^{20}$ définit les modèles d'insertion qui tiennent compte des difficultés du demandeur à entrer dans le champ de la production. Il individualise dans la mesure où il s'élabore dans le cadre de rencontres en face à face, entre un travailleur social représentant "la société", et un "exclu". Cette individualisation déplace désormais la réponse à la question de la pauvreté. Dans les faits, la sortie de "l'exclusion" doit se réaliser par une action pédagogique visant à soutenir l'effort du "pauvre" qui doit s'engager à réaliser des activités dites "d'insertion", c'est-à-dire des activités permettant de renouer des liens avec le monde du travail.

Si les députés socialistes s'inscrivent dans cette pédagogie du retour à l'emploi, les députés de droite ne sont pas unanimes sur cette approche :

"Monsieur le Ministre, cette explication de vote est pour moi l'occasion de rappeler que nous aurions préféré, au groupe R.P.R., que le contrat d'insertion soit la base juridique du versement de l'allocation de revenu minimum, parce que nous pensons que le contrat d'insertion pouvait être le premier échelon du droit au travail qui est prévu dans notre constitution et être assimilable à un contrat de travail." (Jean-Pierre Delalande, R.P.R., débat du 21.11.88, p 2620)

Le contrat d'insertion n'est plus ici un temps de passage mais un contrat de travail. L'activité d'insertion serait alors assimilable à un emploi. L'amalgame entre activité d'insertion et emploi est une façon de dissocier le revenu de ce travailleur d'un nouveau type, du S.M.I.C. ${ }^{21}$ et de l'activité réalisée. Cette conception libérale du R.M.I. permet de ne pas avoir à traiter du paradoxe dans lequel sont les députés socialistes ; en assimilant le R.M.I. à un salaire, il n'est plus nécessaire de se mobiliser pour réintégrer dans le champ économique des populations que les employeurs excluent pour moderniser la production et accentuer la productivité. Cette conception ne signifie pas pour autant le rejet de la notion de responsabilité

20 L'article 36 situe le projet au sein du contrat en ces termes : "(...) un contrat faisant apparaitre :

- tous les éléments utiles à l'appréciation de la situation sanitaire, sociale, professionnelle, financière des intéressés et de leurs conditions d'habitat;

- la nature du projet qu'ils sont susceptibles de former ou qui peut leur être proposé ;

- la nature des facilités qui peuvent leur être offertes pour les aider à réaliser ce projet ;

- le calendrier des démarches et activités d'insertion qu'implique la réalisation de ce projet." Salaire Minimum Interprofessionnel de Croissance. 
individuelle. Comme dans tout contrat de travail, "le bénéficiaire" doit respecter ses engagements s'il veut percevoir son revenu.

Quel que soit le sens donné au contrat, celui-ci est et restera l'expression d'un traitement individuel de la question de la pauvreté. Seule la manière d'éviter la situation paradoxale consistant à dire que l'insertion passe par l'emploi et que l'Etat ne peut pas garantir le droit au travail, diffère. En définissant le contrat d'insertion comme un contrat pédagogique et non comme un contrat de travail, la gauche socialiste ouvre la voie au développement d'activités d'insertion conçues comme des moyens d'aide individuelle à l'insertion et à la création d'une organisation chargée d'orienter les bénéficiaires vers ces activités sensées les métamorphoser. Reste à se demander comment les acteurs chargés de mettre en oeuvre cette loi jouent le jeu de l'insertion.

\section{La réalisation des contrats comme espace de confrontation entre la logique d'insertion et les}

\section{logiques des acteurs.}

Le département est l'espace dans lequel se définit et s'organise le R.M.I.. Qu'ils soient hommes politiques, agents administratifs, travailleurs sociaux ou membres d'associations, leurs interventions auprès de la population bénéficiaire du R.M.I. se délimitent à ce territoire. Au sein de cette aire, différents liens sociaux se tissent autour des espaces de relations pré-définis par la loi (Conseil Départemental d'Insertion ; Commissions Locales d'Insertion, entretiens de réalisation des contrats). Ils constituent une configuration au sens de Norbert Elias, à savoir la figure globale que forme les agents en interdépendance qui "inclut non seulement leur intellect, mais toute leur personne, les actions et les relations réciproques ${ }^{22 " . ~ C h a q u e ~}$ configuration "départementale" prend une forme spécifique en raison des positions des différents agents ou corps d'agents lors de la réalisation de la politique du R.M.I. sur le plan local. Toutes les configurations ont pour vecteur commun la logique d'insertion et constituent au plan national le champ d'exécution de la politique du R.M.I. ${ }^{23}$.

22 Norbert Elias, Qu'est-ce que la sociologie?, La Tour d'Aigues, Ed. de l'Aube, 1991, p 157.

23 Lorsque nous utilisons le terme de champ, nous nous référons directement à ce que Pierre Bourdieu définit comme "un réseau, ou une configuration de relations objectives entre des positions. Ces positions sont définies objectivement dans leur existence et dans leurs déterminations qu'elles imposent à leurs occupants, agents ou institutions, par leur situation (situs) actuelle et potentielle dans la structure de 
En ayant effectué l'étude d'une configuration départementale ${ }^{24}$, et en ayant étudié le contenu d'entretiens entre les assistants sociaux et les bénéficiaires lors de la réalisation des contrats ${ }^{25}$, il a été possible d'étudier la manière dont les différents agents, selon leur position au sein de cet espace, pratique la politique du R.M.I.. Ainsi, les deux acteurs chargés de réaliser les contrats, c'est à dire les travailleurs sociaux et les bénéficiaires, ont des logiques d'action qui ne sont pas nécessairement en conformité avec la logique du champ.

\subsection{Les assistants sociaux sous contrôle politique.}

Les assistants sociaux n'ont pas réalisé les contrats avec empressement. Le rapport de la Commission Nationale d'Evaluation note en 1992 un plafonnement depuis un an à 40\% des bénéficiaires titulaires d'un contrat ${ }^{26}$. En Loire-Atlantique, le pourcentage est plus faible; il est de $31 \%$ en décembre 1990, de 36\% en décembre 1991 et de 33\% en 1992. Dès la première année, les pouvoirs publics se sont inquiétés de cet écart entre le nombre d'allocataires et le nombre de contrats ; Claude Evin, Ministre de la Solidarité, de la Santé et de la Protection sociale incite en 1989 les différents agents à établir des contrats :

"Les données quantitatives n'ont qu'une signification limitée et j'ai souhaité, en effet, depuis le début, que l'on sache prendre du temps plutôt que de multiplier les "contratsbidon". Mais à égalité de contraintes, les résultats sont très variables d'un endroit à l'autre. Un taux de $100 \%$ de contrats signés dans un département ne signifie pas l'excellence, en revanche, un ratio de 3\% pose problème. Il nous faut quand même des indicateurs de cette nature.

la distribution des différentes espèces de pouvoir (ou de capital) dont la possession commande l'accès aux profits spécifiques qui sont en jeu dans le champ, et, du même coup par leurs relations objectives aux autres positions (domination, subordination, homologie, etc.)."( Pierre Bourdieu, Réponses, Paris, Ed. du Seuil, Coll. Libre examen, 1992, p 73).

$24 \quad$ Celle du département de la Loire Atlantique.

25 Plutôt que d'avoir le discours des travailleurs sociaux sur leurs pratiques d'entretien avec les bénéficiaires, il est apparu plus pertinent d'avoir les discours en pratique d'entretien. Il a donc été demandé à des assistants sociaux d'enregistrer leurs rencontres avec des bénéficiaires. Il s'agit de l'entretien où s'élabore le premier contrat écrit et de l'entretien où s'effectue le renouvellement du contrat (entre six et huit mois après le premier).

$26 \quad$ Rapport de la Commission, op.cit., p 308. 
"Le chiffre de 48000 contrats fin juillet, que je jugeais insuffisant à Valence, est passé à 65000 contrats au moins, voire près de 70000, au 30 septembre: progression non négligeable d'environ 20000 contrats en un mois, si on neutralise le mois d'août peu propice aux signatures" $^{27}$.

Ces tensions sont dépendantes des positions entre les agents au sein de la configuration du champ du R.M.I. et plus particulièrement entre les assistants sociaux, les élus et les responsables de services sociaux.

Le nombre des assistants sociaux est passé de 18921 en $1970^{28}$ à 36000 en $1990^{29}$. A cette évolution quantitative liée au développement de la protection sociale, s'est adjoint une modification des relations hiérarchiques entre assistants sociaux polyvalents de secteur et leurs employeurs. Avant les lois sur la décentralisation, les assistants sociaux polyvalents de secteur étaient employés par les services déconcentrés de l'Etat (Directions Départementales des Affaires Sanitaires et Sociales), par les Mutualités Sociales Agricoles, par les Caisses d'Allocations Familiales et par des Centres Communaux d'Action Sociale. A partir de 1983, l'employeur principal devient le Président du Conseil Général. Les conventions passées avec les M.S.A. et les C.A.F. ont été progressivement rompues. En Loire-Atlantique, les assistants sociaux polyvalents de secteur sont tous sous la responsabilité hiérarchique du Président du Conseil Général excepté pour deux circonscriptions ${ }^{30}$ (une circonscription est sous la responsabilité de la C.A.F. ; l'autre sous celle du C.C.A.S. de Nantes). Ce changement d'employeurs conséquent à la critique de l'intervention de l'Etat, n'est pas sans effet sur les relations entre les différents niveaux de la hiérarchie administrative.

27 Interview de Claude Evin, "Claude Evin : mes quatre vérités sur l'insertion", Actualités sociales Hebdomadaires, $\mathrm{n}^{\circ} 1662,27.10 .89, \mathrm{p} 9$.

28 Données recueillies dans le cadre d'une enquête de l'INSERM par Lucien Brams et Nicole Courtecuisse, Contribution à la sociologie d'une profession : les assistants de service social en France, Paris, rapport I.N.S.E.R.M. 1072, 1970, p 13.

29 Données sociales, Paris, I.N.S.E.E ,1990, p 542.

30 Le département comporte 17 circonscriptions et 250 assistants sociaux de polyvalence sont employés par la Direction des lnterventions Sanitaires et Sociales. 
En Loire-atlantique, les responsables des services sociaux départementaux sont en relation étroite avec la Présidente de la Commission des Affaires Sociales du Conseil Général, chargée d'assurer la mise en oeuvre des politiques sociales de lutte contre l'exclusion relevant de la responsabilité partagée entre l'Etat et le Département.

La loi sur le R.M.I. est la première politique qui engage ainsi les Conseils Généraux et par voie de conséquence les responsables des services sociaux du département. Ces agents administratifs deviennent alors les conseillers techniques des élus politiques. Ils sont à leur disposition pour mettre en place les structures prévues par les textes, pour organiser les modalités de fonctionnement, pour préparer les réunions du Conseil Départemental d'Insertion. Reconnus dans leur compétence pour organiser le dispositif du R.M.I., ils sont parallèlement contraints de satisfaire les exigences du pouvoir politique local qui tient à rendre visible l'engagement du Conseil Général dans la politique de lutte contre l'exclusion. Les deux indicateurs reconnus par les conseillers généraux et par les responsables de services pour apprécier cet engagement sont le nombre de contrats réalisés et l'évolution du nombre d'entrée et de sortie du dispositif ${ }^{31}$.

Face aux faibles pourcentages de contrats, les responsables des services sociaux et les élus se retournent vers les salariés chargés de réaliser les contrats et d'effectuer les accompagnements sociaux, à savoir les assistants sociaux polyvalents de secteur. La pression exercée à leur encontre prend différentes formes. Il peut s'agir de courriers rappelant la nécessité de réaliser les contrats dans un délai se rapprochant des limites imposées par la $1 \mathrm{loi}^{32}$, de réunions de service ou de réunions avec les membres de la C.L.I..

Les résistances des assistants sociaux peuvent s'expliquer par deux raisons :

- Avec la décentralisation et la mise en place des politiques de lutte contre l'exclusion, les assistants sociaux perdent une part de leur liberté d'exercice. Ils n'ont plus autant la possibilité de définir eux-mêmes ce qui est nécessaire pour le client ${ }^{33}$ demandeur d'une aide. L'évaluation de la situation du bénéficiaire par l'assistant social est désormais une demande des responsables du service. Si l'assistant social ne prend pas rendez-vous avec le bénéficiaire, le nombre de contrats réalisés tend à stagner.

31 Lors des bilans réalisés par les responsables de service et présentés au C.D.I., la politique du R.M.I. est évaluée selon ces deux indicateurs.

32 Le contrat doit être signé dans les trois mois suivant le versement de l'allocation.

33 La présence de ce terme dans le vocabulaire des assistants sociaux a été analysé dans un ouvrage très controversé de Jeanine Verdès-Leroux, Le travail social, Paris, Ed. de Minuit, 1978, 273 p. 
- Par ailleurs, les assistants sociaux sont directement confrontés au paradoxe lié à la loi sur le R.M.I.. Ils rencontrent des agents en vue d'élaborer des "actions d'insertion" alors qu'ils n'ont pas la certitude que les bénéficiaires aient un emploi au terme de ce "parcours". S'ils ne critiquent pas la démarche du contrat d'insertion, ils évoquent leur impuissance à conduire les bénéficiaires vers le retour à l'emploi ${ }^{34}$.

Les relations entre les différents agents institutionnels ont été évoquées dans le rapport de la Commission nationale: "Le processus de décentralisation de l'action sociale, en faisant dépendre les travailleurs sociaux des élus du département, les a déstabilisés. (...) Dans la mesure où c'est la C.L.I. qui valide le contrat d'insertion, le travailleur social peut se sentir dépossédé par des institutions qui contestent son diagnostic et lui font perdre sa relation privilégiée avec le bénéficiaire. (...) Enfin - et c'est le problème le plus fondamental - la culture et la qualification des travailleurs sociaux de secteur, qui sont pour l'essentiel des assistantes sociales ne correspondaient plus que partiellement à la demande de travail des personnes relevant de la "nouvelle pauvreté" sociaux caractérise parfaitement les rapports de force entre les divers gestionnaires locaux du R.M.I., d'un côté les élus et responsables de services sociaux souhaitant avoir une plus grande maîtrise de l'application du R.M.I., de l'autre les assistants sociaux utilisant les ressources liées à leur position pour affirmer leur volonté de conserver la maîtrise de l'accompagnement social des personnes s'adressant à eux ${ }^{36}$.

Cependant, si les assistants sociaux ne réalisent pas autant de contrats que les responsables et les élus le souhaiteraient, cela est plus une résistance aux modifications des tâches et des relations institutionnelles que provoque la mise en place du R.M.I., qu'une critique de la politique en elle-même. Tous les agents inscrits dans le champ d'exécution de la politique du R.M.I. s'accordent pour focaliser l'insertion sur les bénéficiaires. Ces derniers ne sont pas passifs face à ce coup de force qui consiste à penser que la lutte contre la pauvreté relève d'une prise en charge individuelle.

\footnotetext{
34 Les différentes enquêtes menées dans le cadre de la Mission Interministérielle Recherche Expérimentation ont évoqué cet aspect. A titre d'illustration, la synthèse de la recherche menée en Gironde note que les travailleurs sociaux ont "conscience que le dispositif, en raison de la diversité des populations concernées, ne constitue pas une réponse adaptée à tous les cas ; les résultats dans le domaine de l'insertion professionnelle paraissent plus hypothétique" (MIRE,Gironde, p 14).

35 Rapport de la Commission, T1, op. cit., pp 346-348.

36 Voir article du Monde du 20.9.93 intitulé :"Assistantes sociales contre élus locaux".
} 


\section{2 - Les bénéficiaires face à la logique d'insertion.}

L'acceptation du rendez-vous proposé au bénéficiaire ${ }^{37}$ pour élaborer un "contrat d'insertion" signifie pour le travailleur social un accord ${ }^{38}$ sur la finalité de l'entretien. Le contrat étant dit "d'insertion", le bénéficiaire acceptant la rencontre, l'assistant social présuppose que la personne accepte la logique du champ. Il est situé a priori comme un agent désirant (re)trouver un emploi mais dont les difficultés spécifiques limitent ses chances d'accès au marché. Ce rapport entre les deux locuteurs définit la procédure d'entretien. Pour le travailleur social, l'entretien doit lui permettre de qualifier la situation du bénéficiaire sur une échelle de difficultés afin d'y apporter une solution.

\section{A- Une procédure centrée sur le manque.}

La première phase de la rencontre, caractérisée par des réitérations et des interrogations formulées par le travailleur social, est consacrée à une exposition des situations familiale, scolaire, professionnelle et de leurs évolutions par le bénéficiaire. Le thème central est l'activité professionnelle passée (forme de travail et d'emploi, rapport au travail et à l'emploi) ; il représente sur l'ensemble des entretiens étudiés $51 \%$ des tours de parole du travailleur social pendant cette phase d'investigation. L'objectif du questionnement est de repérer les "inadaptations", les "handicaps", les "difficultés" de la personne.

(Entretien entre un assistant social et Pierre, âgé de 43 ans, vivant dans un squat, sans travail salarié depuis 1978 tout en ayant exercé des travaux au noir jusqu'en 1988).

Assistant social : Donc vous êtes bénéficiaire du R.M.I.. On considère un petit peu que la contrepartie de cette allocation que vous percevez, c'est de souscrire à un contrat d'insertion pour vous permettre de vous réinsérer soit professionnellement soit socialement,

37 Toutes ces rencontres ont été mises en place à l'initiative du travailleur social qui avait préalablement précisé la finalité (réaliser le contrat d'insertion dans lequel apparaît le "projet d'insertion") et demandé l'accord de la personne pour que l'entretien soit enregistré.

$38 \quad$ Cet accord préalable est ce que H-Paul Grice appelle le principe de coopération : "Nos échanges de paroles ne se réduisent pas en temps normal à une suite de remarques décousues, et ne seraient pas rationnels si tel était le cas. Ils sont le résultat, jusqu'à un certain point au moins, d'efforts de coopération; et chaque participant reconnaît dans ces échanges (toujours jusqu'à un certain point) un but commun ou un ensemble de buts, ou au moins une direction commune acceptée par tous" (H-Paul Grice, "Logique et

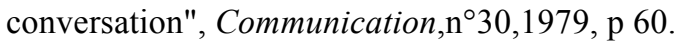


puisque bon, vous êtes un petit peu déconnecté du monde du travail et du monde social en général, quoi hein! Alors, moi je vous ai reçu; disons que, si vous voulez, la finalité de tout ce qu'on va vous dire, on va essayer de dégager un problème pour que vous trouviez soit une forme de réinsertion professionnelle, soit sociale, soit les deux. Et tout ça pour vous faire tendre... Si vous voulez, moi je suis content le jour où je ne vois plus les gens, ça veut dire qu'ils sont réinsérés".

Cette prise de position que l'on retrouve dans l'ensemble des entretiens recueillis, définit les positions. Le travailleur social se place ici comme le guide, le conseiller d'un bénéficiaire perçu comme un individu ayant des problèmes qu'il ne peut pas résoudre seul. Le seul manque qui ne soit pas situé sur le registre de la responsabilité individuelle, est le manque de travail salarié. Les failles de la personne accroissent les difficultés à retrouver un emploi mais n'en sont pas la cause. Elle est présupposée avoir un rapport positif à l'emploi. En effectuant cette distinction, les travailleurs sociaux reconnaissent le chômage comme une fatalité du destin, et construisent une définition du status ${ }^{39}$ du bénéficiaire du R.M.I. ; il s'agit d'agents qui, à la différence des chômeurs indemnisés subissant un contexte socio-économique défavorable, sont également porteurs de stigmates qui réduisent leur possibilité d'accès au monde du travail.

Cette investigation approfondie sur des manques psychosociaux doit permettre, dans une seconde phase de l'entretien, d'élaborer un projet d'insertion. L'assistant social est le seul à être capable de repérer les écarts normatifs des agents, puis de les désigner :

(Entretien entre une assistante sociale et Aïcha, âgée de 37 ans, d'origine tunisienne et demeurant en France depuis son mariage en 1974, divorcée, ayant effectué des stages comme vendeuse, sans emploi).

Assistante sociale : Bon, moi je repère quand même des facteurs un peu défavorables pour vous, c'est le peu d'expérience professionnelle, c'est un niveau scolaire pas très élevé, c'est aussi une difficulté à manier le langage, notamment quand on est dans le cadre de la

$39 \quad$ Nous reprenons la distinction de Dominique Schnapper entre statut au sens juridique et statut au sens sociologique (désigné par "status") qui se caractérise comme "un ensemble plus ou moins systématisé et relativement fixe de comportements, qu'on peut attendre, d'une part, d'un individu placé dans une situation donnée, d'autre part de la société à l'égard de cet individu, et qui sont reconnus comme légitimes par l'individu et la société" (Dominique Schnapper, 1989, "Rapport à l'emploi, protection sociale et status sociaux", in Revue française de sociologie, Paris, janvier-mars, XXX-1,1989, p 3). 
vente. Mais en même temps, ce que je trouve intéressant, ce que je trouve positif, c'est une certaine stabilité dans votre existence".

La désignation a pour objectif la reconnaissance par les bénéficiaires de leur identité sociale spécifique. Cette acceptation des manques, qui constitue la troisième phase de l'entretien, est nécessaire pour que le travailleur social puisse consigner le projet sur le formulaire administratif transmis à la Commission Locale d'Insertion. Cependant faire accepter ne va pas de soi ; les bénéficiaires ne sont pas passifs. La force d'imposition de l'assistant social, lors de l'entretien, est fonction des prises de position du bénéficiaire.

\section{B-Les conditions d'acceptation du manque par le "bénéficiaire".}

Les solutions pour l'insertion étant pensées par l'assistant social à partir des données montrant l'existence d'un comportement ou d'une situation perçu comme anormal, le retour immédiat vers le monde du travail n'est pas envisagé. Il est nécessaire de proposer à la personne des activités qualifiées, selon le degré des carences relevées, d'insertion sociale, d'insertion sociale et professionnelle ou d'insertion professionnelle. Dès lors, l'attente du travailleur social est d'avoir à sa disposition, non pas des offres d'emploi, mais un nombre suffisant d'activités d'insertion à proposer. Cependant, les solutions qu'il propose sont dépendantes de ce que le bénéficiaire a bien voulu dire lors des entretiens et doivent lui convenir. L'acceptation des solutions par le bénéficiaire n'est pas aléatoire. Elle dépend de ses rapports à l'emploi et aux services sociaux qui se sont structurés au cours de sa trajectoire individuelle et sociale.

En référence aux catégories d'utilisateurs des services sociaux définies par Serge Paugam ${ }^{40}$, les bénéficiaires adhérant aux "efforts" demandés par le travailleur social pour retrouver un emploi, sont des personnes faisant l'expérience d'une assistance différée et des bénéficiaires marginaux cherchant à conjurer leur exclusion. Ils peuvent accepter plus aisément les propositions du travailleur social dans la

\footnotetext{
$40 \quad$ Serge Paugam, La disqualification sociale - essai sur la nouvelle pauvreté-, Paris, Ed. P.U.F.,1991, 254 p.
} 
mesure où ils reconnaissent leurs difficultés et souhaitent éviter la situation d'assistance ou d'exclusion. Toute activité proposée qu'il s'agisse de rechercher un logement, d'améliorer le budget ou d'effectuer un stage de remise à niveau, représente pour eux une étape préalable au retour à l'emploi. De ce fait, le contrat est un écrit où l'assistant social formule le projet de telle manière qu'il apparaisse comme celui du bénéficiaire, soutenu par le travailleur social.

Certains bénéficiaires ont une adhésion plus relative. C'est le cas des assistés installés ou revendiquants qui ont perdu tout espoir de trouver un emploi, et qui ont pris l'habitude de bénéficier des différentes prestations dans lesquelles il n'est pas demandé de contrepartie (Allocation de Parent Isolé ; Allocation mensuelle de l'Aide Sociale à l'Enfance par exemple). Pour cette population, le R.M.I. n'est pas nécessairement avantageux, même s'il est une sécurité financière. La contrainte à l'effort d'insertion contrarie leurs pratiques de l'assistance. La négociation avec le travailleur social est alors plus difficile. Dans la mesure où ils ne souhaitent pas rompre la relation, ils doivent utiliser des arguments susceptibles de satisfaire les attentes du travailleur social sans compromettre leur situation d'assistés. Ayant une bonne connaissance de ce qu'il est nécessaire de dire pour perdre le moins possible, ils savent montrer les difficultés prouvant leurs incapacités à s'engager dans des actions visant directement un retour à l'emploi.

Lorsque les bénéficiaires s'opposent aux propositions du travailleur social, celui-ci renforce sa position d'agent chargé de définir la contrepartie au versement du revenu, sans pour autant leur imposer ses "projets". Pour cela, il peut rappeler que la C.L.I. peut ajourner le contrat, utilisant cette instance comme lieu de la censure pour préserver sa relation au bénéficiaire. S'il ne parvient pas au cours des entretiens à imposer sa solution, l'écrit du contrat reprend le projet du bénéficiaire. Cependant, le style du texte devient un moyen ultime pour contraindre la personne à jouer le jeu de l'insertion. Il n'est plus question d'appuyer les propositions. Le contrat est laconique.

De tels différends apparaissent lorsque le bénéficiaire a un rapport à l'emploi positif et ne souhaite pas autre chose qu'un emploi, ou a un rapport à l'emploi négatif que l'assistant social ne peut pas justifier au regard des investigations menées. En reprenant les catégories de Serge Paugam, ces comportements de refus correspondent à deux catégories d'utilisateurs des services sociaux. Les bénéficiaires entrant dans la catégorie des fragiles, souhaitant ne pas s'installer dans l'assistance et vivant difficilement le fait de ne pas avoir un emploi, peuvent avoir une attitude qui consiste à ne pas accepter un contrat qui ne soit pas 
directement lié à l'emploi. A partir des résultats de l'enquête du C.E.R.C., Serge Paugam constate que "ces allocataires du R.M.I. établissent une hiérarchie dans les mesures d'aide à l'emploi qui leur sont proposées. Celles qui se rapprochent le plus du "vrai" travail et qui reposent sur un contrat signé avec un employeur, et non pas avec une instance représentant la collectivité, sont un signe de reconnaissance sociale de leurs qualifications ou de leurs capacités à exercer une activité professionnelle au même titre ou presque que les autres travailleurs ${ }^{41}$ ". Cependant, même si le R.M.I. ne compense pas leur sentiment d'insatisfaction, ils le reconnaissent comme étant un moyen de mieux organiser leur vie quotidienne. De ce fait, même s'ils sont contraints de se plier aux exigences du champ, ils ne cherchent pas à rompre avec les services sociaux.

La rupture avec les services sociaux peut apparaître avec les marginaux organisés. Alors que le revenu constitue pour eux un fixe, ils ne sont pas nécessairement disposés à dévoiler des aspects de leur histoire ou à s'engager dans des actions qui s'inscrivent dans un espace et un temps social qui n'est plus le leur. Refusant l'éventualité de s'inscrire dans une perspective lointaine de retour à l'emploi, ils imposent aux travailleurs sociaux leurs propres souhaits, ce qui n'est pas sans risque. Si les propositions n'entrent pas dans la logique du champ d'exécution de la politique du R.M.I., la C.L.I. peut ajourner le contrat présenté dans lequel le travailleur social ne s'est pas directement impliqué. Face au coup de force, certains bénéficiaires ne peuvent pas se maintenir dans le champ d'exécution de la politique du R.M.I..

La contractualisation est un temps qui institue le caractère individuel du processus d'insertion. Par le repérage des difficultés justifiant "l'inemployabilité" du bénéficiaire, se légitime l'existence d'un nouveau champ, celui de l'activité d'insertion, dont la principale caractéristique est d'accueillir objectivement ceux qui ne peuvent plus prétendre à un emploi dans le cadre de l'économie de marché mais qui, subjectivement sont ceux qui ont besoin d'une aide pour avoir la possibilité de trouver un emploi "classique" dans un futur indéfini. En ne définissant pas d'emblée le bénéficiaire comme un chômeur sans indemnité, la question de la résolution du chômage structurel glisse subtilement vers la question du développement d'une "nouvelle" économie, l'économie solidaire, qui offrirait des nouveaux emplois à ceux qui ne peuvent en avoir en raison

41 Serge Paugam, 1991, Atouts et difficultés des allocataires du R.M.I. - rapport final -, Centre d'Etude des Revenus et des Coûts, Paris, Ed. La documentation française, Nº102, 3ème trim 1991,p 96. 
de leur incapacité à s'adapter aux transformations de la société. Ce glissement s'explique par le postulat qui consiste à centrer la question de la pauvreté sur les individus.

$\circ$

$\circ \quad \circ$

La centration sur l'individu est ce qui permet à la fois de tenir un discours sur l'impossibilité du plein emploi et de mettre en place des actions d'insertion visant à terme le retour à l'emploi. En effet, s'il n'est plus possible d'offrir un emploi à toute personne au sein même de l'économie de marché, cela ne concerne pas tout le monde. Sont visées les personnes ayant une carence réduisant leur capacité d'adaptation aux nécessités de souplesse du marché de l'emploi. Pour celles-ci, il devient légitime de concevoir non pas un emploi mais une activité dans le cadre d'une "économie solidaire". La pratique du R.M.I. consiste à orienter les bénéficiaires vers les lieux adéquats après avoir réalisé une investigation des carences expliquant l'impossibilité d'un retour immédiat sur le marché de l'emploi et après avoir élaboré un contrat concrétisant l'engagement des bénéficiaires. Cette organisation n'est pas manichéenne. Elle est un effet d'une des variantes d'une même pensée, le solidarisme, elle-même indissociable de la structure de notre système socio-économique. L'idée de progrès fondée sur la valeur sociale du travail, l'approche de la société comme un ensemble de relations interindividuelles engageant de façon contractuelle les responsabilités individuelles et collectives, le processus d'individualisation, sont liés au développement de l'économie de marché. Mais il serait vain d'ignorer que ce développement n'est pas consensuel. Il s'effectue dans des rapports de force entre différents groupes sociaux. "Dépaysannisation, désouvriérisation, exclusion. L'économie de marché restructure sans cesse et déstructure aussi. Ceux qui engagent les frais ne sont pas forcément ceux qui payent ${ }^{\prime 42}$. Ce propos de M. Verret a l'intérêt de présenter la question sociale sous un jour différent. Comment les fruits du progrès peuvent-ils être partagés par le plus grand nombre si ceux qui engagent les frais ne s'impliquent pas dans la recherche de solutions ou ne sont pas contraints par les pouvoirs politiques de s'y impliquer?

42 Verret. M., "Statistiques de classes" in Politis-La revue, Paris, 4ème trim 1993, pp 39-42. 
Alain THALINEAU

LAST-VST 